\title{
Thyroid Dysfunction and Its Influence in Growth Velocity in Children with Down Syndrome: A Retrospective Study
}

\author{
${ }^{1}$ Vidhya Vishwanathan, ${ }^{2}$ Subramanian Sethuraman, ${ }^{3}$ Shanthi Balaji, ${ }^{4}$ VS Kalai Selvi, ${ }^{5}$ AJ Manjula Devi
}

\begin{abstract}
Introduction: Down syndrome is one of the common chromosomal anomaly which has various endocrinological abnormalities, of which thyroid dysfunction is very common. When Down syndrome and thyroid dysfunction are present together, it leads to amplification of the clinical problem.
\end{abstract}

Aims and objectives: With this background, it was aimed to study the incidence of thyroid dysfunction in Down syndrome in pediatric ( $<12$ years) age group and measure the growth velocity before and after initiation of treatment.

Materials and methods: It is a retrospective analysis, which includes 50 children of Indian origin with Down syndrome between the age group of 1 and 12 years from neurodevelopmental clinic (multicentric) who met the phenotypic criteria for establishing the diagnosis of Down syndrome. The thyroid reports and ultrasound reports of thyroid gland for children with hypothyroidism were extrapolated and analyzed. Birth history, age of diagnosis of thyroid disorder, initiation of treatment, normalization of thyroid function, and anthropometric measurements before and 1 year after initiation of treatment were noted.

Results: In this study, thyroid disorder (hypothyroidism) was found in $12(24 \%)$ children, of which 7 (14\%) were diagnosed at the age group of 0 to 1 year and $5(10 \%)$ were diagnosed at the age of 1 to 12 years. The mean height in percentile was $9.92 \pm 5.63$ before and $43.75 \pm 12.68$ after supplementation of these children with L-thyroxine. None of the children had hyperthyroidism $(p=0.0001)$.

Conclusion: As mental and physical retardation are common denominators in both Down syndrome and hypothyroidism, coexistence of both the conditions would lead to further developmental delay in terms of mental as well as physical health. Annual screening of thyroid function should be employed in these children to diagnose the problem at the earliest and initiate treatment, thereby improving the quality of life.

Keywords: Down syndrome, Endocrinology, Growth velocity, Hypothyroidism, Thyroid disorder.

\footnotetext{
${ }^{1}$ Postgraduate Student, ${ }^{2}$ Consultant Pediatrician, ${ }^{3} \mathrm{Head}$ ${ }^{4,5}$ Professor

1,3-5 Department of Biochemistry, Sree Balaji Medical College \& Hospital, Chennai, Tamil Nadu, India

${ }^{2}$ Department of Pediatrics, Smrthi Health Care, Chennai, Tamil Nadu, India

Corresponding Author: Vidhya Vishwanathan, Postgraduate Student, Department of Biochemistry, Sree Balaji Medical College \& Hospital, Chennai, Tamil Nadu, India, Phone: +919677914645, e-mail: vidhuvishwa1@gmail.com
}

How to cite this article: Vishwanathan V, Sethuraman $S$, Balaji S, Selvi VSK, Devi AJM. Thyroid Dysfunction and Its Influence in Growth Velocity in Children with Down Syndrome: A Retrospective Study. Indian J Med Biochem 2018;22(1):6-9.

Source of support: Nil

Conflict of interest: None

\section{INTRODUCTION}

Down syndrome/Trisomy 21 is the most common chromosomal anomaly with an incidence of 1 in 800 live births, and it is also an added factor for growth retardation and intellectual disabilities. ${ }^{1}$ Down syndrome patients present with various birth defects. Down syndrome has an increased risk of developing endocrinal abnormalities, especially thyroid disorders. It consists of a constellation of clinical signs and symptoms and associated with various forms of thyroid dysfunction (congenital/acquired). ${ }^{2}$ Hypothyroidism accounts for about $28 \%{ }^{3}$ The additive effects of both comorbid conditions lead to further amplification of the clinical problems in these children, the symptoms of which are often mistaken for the natural course of the disease. ${ }^{3}$ The prevalence of hypothyroidism is more compared with hyperthyroidism. The prevalence of congenital hypothyroidism is 30 times more in newborn with Down syndrome than normal newborns. ${ }^{4}$ This is a treatable cause of mental retardation, thus early detection and treatment are essential in order to maximize cognitive abilities in this already impaired population. ${ }^{4}$

\section{AIM}

The study was aimed to see the influence of thyroid dysfunction in growth velocity in children with Down syndrome. With the objectives to estimate the incidence of thyroid disorder in the study group, ultrasonography of the thyroid gland in these children to see whether there is any dysplasia, to see the age-wise distribution, and the influence of thyroid dysfunction in growth velocity before and after initiation of treatment with L-thyroxine in the children with Down syndrome.

\section{MATERIALS AND METHODS}

It is a retrospective analysis which includes 50 children of Indian origin with Down syndrome between the age 


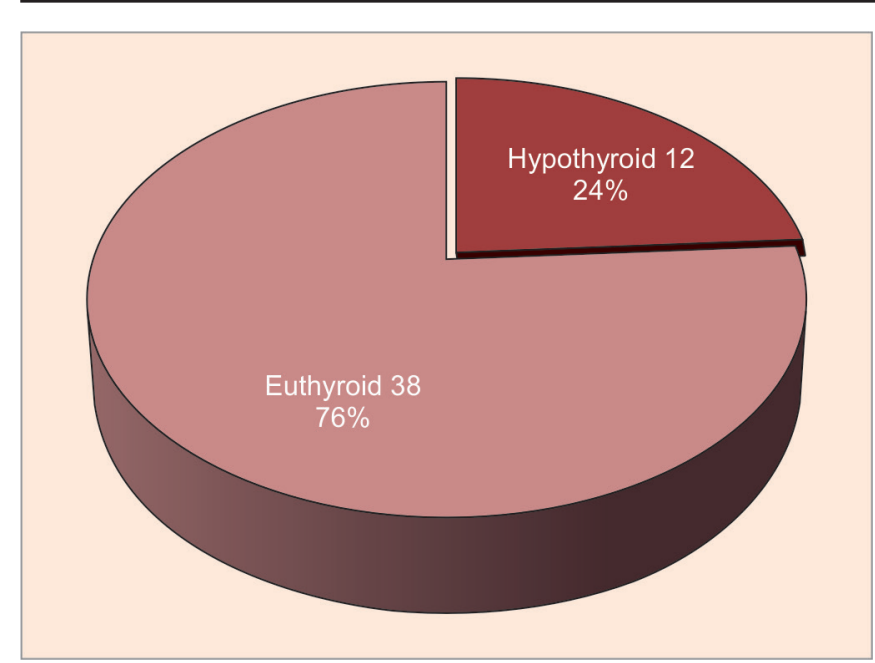

Graph 1: Incidence of thyroid disorder in the study group

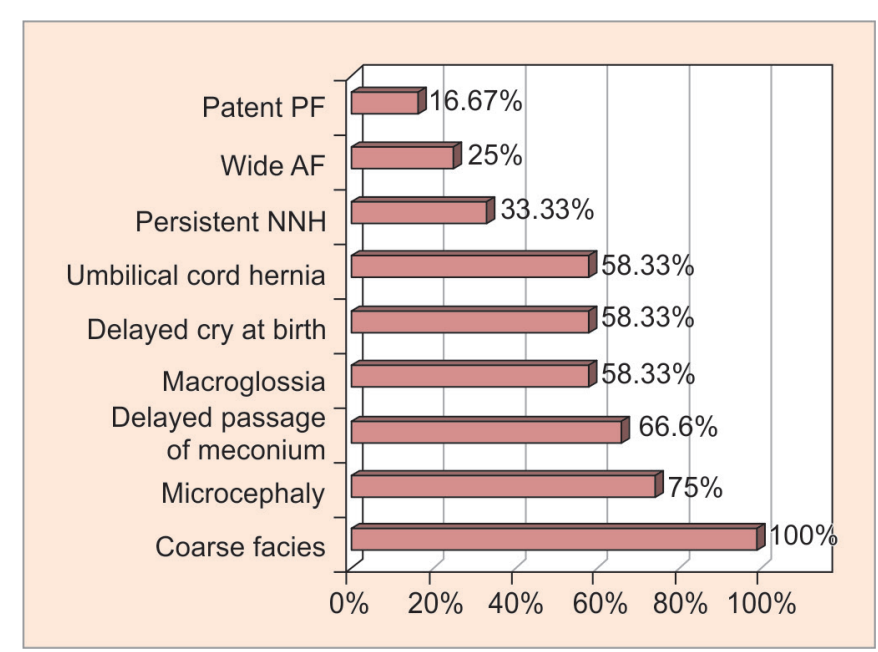

Graph 2: Clinical presentation of children with thyroid disorder at birth group of 1 to 12 years from neurodevelopmental clinic (multicentric), who met the phenotypic criteria for establishing the diagnosis of Down syndrome. The study was conducted at the Department of Biochemistry, Sree Balaji Medical College \& Hospital, Chennai, Tamil Nadu, India, during February 2016 to July 2016. Informed parental consent was obtained. Thyroid reports and ultrasound reports of thyroid gland for children with hypothyroidism were extrapolated and analyzed. A diagnosis of hypothyroidism was made based on a combination of raised serum concentration of thyroid stimulating hormone (TSH) and/or low serum concentration of free thyroxine. Birth history, age of diagnosis of thyroid disorder, initiation of treatment, normalization of thyroid function, and anthropometric measurements before and 1 year after initiation of treatment with L-thyroxine were noted. Maternal thyroid status and weight of the child were not included in the study. Statistical analysis was done using the Statistical Package for the Social Sciences Version 18.

\section{Inclusion Criteria}

- Diagnosis confirmed by karyotyping

- Detailed hospital record maintained

- Birth to 12 years of age.

\section{Exclusion Criteria}

- Down syndrome $>12$ years of age

- Multiple congenital anomalies.

\section{RESULTS}

In the current study, 12 children (i.e., incidence of $24 \%$ ) were found to have thyroid disorder. All the 12 children had hypothyroidism and none of the current study population was found to have hyperthyroidism (Graph 1).
Out of 50 children with Down syndrome included in the study population, $28(56 \%)$ were males and $22(44 \%)$ were females. Seven out of 28 male and 5 out of 22 female children were found to have hypothyroidism.

Ultrasound reports of these 12 children with hypothyroidism were analyzed, which showed hypoplasia of the thyroid gland in 4 children that accounts for $33.33 \%$ in the study population.

Out of these 12 children with hypothyroidism, the following clinical presentation was observed in birth—delayed cry at birth, umbilical cord hernia (58.33\%), macroglossia (58.33\%), delayed passage of meconium (66.67\%), persistent neonatal hyperbilirubinemia (33.33\%), microcephaly $(75 \%)$, coarse facies $(100 \%)$, wide anterior fontanelle $(25 \%)$, and patent posterior fontanelle (16.67\%) (Graph 2).

The incidence of hypothyroidism was more $(14 \%)$ in 0 to 1 year and $10 \%$ in the age of 1 to 12 years (Graph 3).

The height/length of children at the time of diagnosis of hypothyroidism and 1 year after treatment

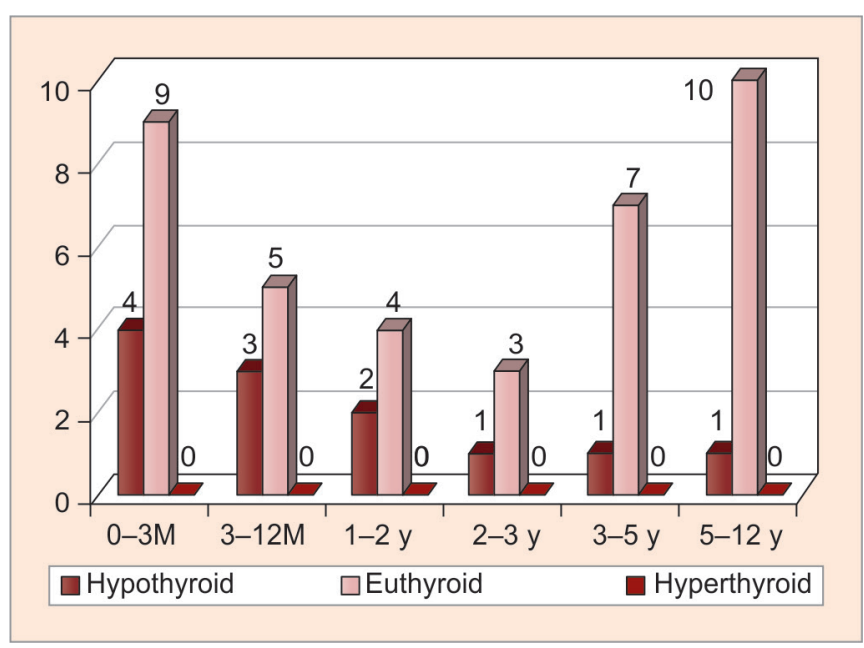

Graph 3: Age-wise distribution of thyroid disorder in children with Down syndrome 


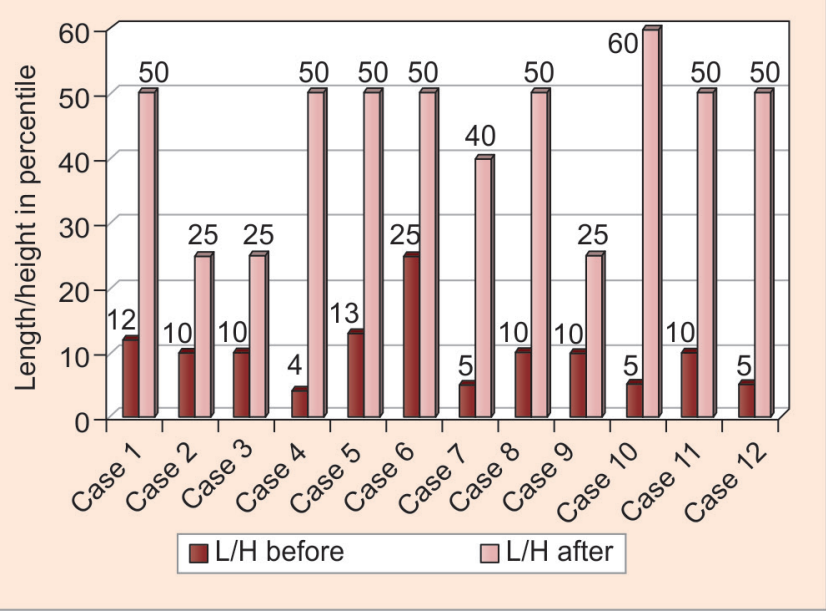

Graph 4: Growth percentile before and after initiation of treatment in children with hypothyroidism

with L-thyroxine were noted in the study subjects. The individual values were compared with standard growth charts for Down syndrome provided by the Centers for Disease Control and Prevention. The mean height in percentile was $9.92 \pm 5.63$ before and $43.75 \pm 12.68$ after supplementation ( $\mathrm{p}=0.0001$, Graph 4 ).

\section{DISCUSSION}

There is a wide variation in the data regarding the incidence of thyroid dysfunction among Down syndrome. ${ }^{5}$ In the current study, prevalence of $24 \%$ was observed. Similar prevalence was noted by Pierce et al. ${ }^{6}$

There was no sex difference observed in the current study. This was comparable to the study done by Karlsson et al, ${ }^{7}$ where they did not find any sex difference. The authors also observed half of the patients with hypothyroidism acquired the condition before the age of 8 years, but only one of them displayed thyroid autoantibodies at diagnosis. Most patients who developed hypothyroidism after this age had thyroid autoantibodies.

The prevalence of $14 \%$ in the age group of 0 to 1 year in the present study could well be taken as a reflection of the prevalence of congenital hypothyroidism, which is consistent with the study done by van Trotsenburg et al. ${ }^{8}$ They observed mild hypothyroid state in newborns with Down syndrome, which supports the existence of Down syndrome-specific thyroid disorder. Hypothyroidism is due to delayed maturation of hypothalamopituitary axis, which is attributed to delayed switching over somatomedins from fetal to adult form. Thyroid stimulating hormone receptor or their signaling proteins (G protein) could be another possible mechanism.

Hypothyroidism was observed in $10 \%$ of children between 1 and 12 years. This acquired form of hypothyroidism is usually associated with thyroid antibodies.
However, thyroid antibodies were not measured in the current study as it was not included in the study protocol.

Hyperthyroidism was not observed in any of the cases included in the study, though a few cases were reported 6.5 per thousand in a study conducted by Alberto GodayArno et al, ${ }^{9}$ case reports by Szatkowska et al, ${ }^{10}$ and $2.1 \%$ as detected by Unachak. ${ }^{11}$

The mean height in percentile was $9.92 \pm 5.63$ before and $43.75 \pm 12.68$ after supplementation $(\mathrm{p}=0.0001)$. Studies have shown that untreated hypothyroidism may affect the growth as well as psychomotor development. ${ }^{5}$ Initiating treatment at the earliest could improve the growth velocity.

The results of a study done by Rashid et $\mathrm{al}^{12}$ show raised levels of TSH and antithyroperoxidase in duodenal switch patients. Subclinical hypothyroidism is found in significant number of patients. Assessment of thyroid function in patients of Down syndrome is recommended at regular intervals.

The American Academy of Pediatrics (AAP) recommends thyroid screening at birth, 6, 12 months, and then annually. Early screening is recommended for the early diagnosis and planning of the treatment strategies in order to provide an improved quality of life. ${ }^{13,14}$

\section{CONCLUSION}

As mental and physical retardation are common denominators in both Down syndrome and hypothyroidism, coexistence of both the conditions would lead to further developmental delay in terms of mental as well as physical health. Annual screening of thyroid function should be conducted in these children to diagnose the problem at the earliest and initiate treatment, thereby improving the quality of life.

\section{FUTURE SCOPE}

- Further studies investigating thyroid antibodies in these patients are needed.

- Since this is a retrospective study, cognitive abilities are not accessed.

\section{LIMITATIONS OF THE STUDY}

Since it is a retrospective study, cognitive abilities of the study are not accessed. Both Down syndrome and thyroid disorders can decrease the cognitive ability of the child.

\section{REFERENCES}

1. Gardiner KJ. Molecular basis of pharmacotherapies for cognition in Down syndrome. Trends Pharmacol Sci 2010 Feb;31(2):66-73. 
2. Myrelid A, Jonsson B, Guthenberg C, von Dobeln U, Anneren G, Gustafsson J. Increased neonatal thyrotropin in Down syndrome. Acta Paediatr 2009 Feb;98(6):1010-1013.

3. Cebeci AN, Güven A, Yıldız M. Profile of hypothyroidism in Down's syndrome. J Clin Res Pediatr Endocrinol 2013 Jun;5(2):116-120.

4. McGowan S, Jones J, Brown A, Reynolds L, Leyland K, Charleton P, Rahim M, Mansor M, Ritha S, Donaldson M, et al. Scottish Down Syndrome Thyroid Screening Group. Capillary TSH—screening programme for Down's syndrome in Scotland, 1997-2009. Arch Dis Child 2011 Dec;96(12): 1113-1117.

5. Lebel EW, Tenenbaum A, Malkiel S, Kastiel Y, Abu-Libdeh A, Zangen D. Low-normal FT4 and subclinical hypothyroidism may have a detrimental clinical effect in Down syndrome. Horm Res Paediatr 2011;76(Suppl 2):46-47.

6. Pierce MJ, LaFranchi SH, PinterJD. Characterization of thyroid abnormalities in a large cohort of children with Down syndrome. Horm Res Paediatr 2017;87(3):170-178.

7. Karlsson B, Gustafsson J, Hedov G, Ivarsson S, Anneren G. Thyroid dysfunction in Down's syndrome: relation to age and thyroid autoimmunity. Arch Dis Child 1998 Sep;79(3): 242-245.
8. van Trotsenburg AS, Vulsma T, Van Santen HM, Cheung W, de Fielder JJ. Neonatal screening thyroxine concentration in Down syndrome newborns. J Clin Endocrinol Metab 2003 Apr;88(4):1512-1515.

9. Goday-Arno A, Cerda-Esteva M, Flores-Le-Roux JA, Chillaron-Jordan JJ, Corretger JM, Cano-Pérez JF. Hyperthyroidism in a population with Down syndrome (DS). Clin Endocrinol 2009 Jul;71(1):110-114.

10. Szatkowska M, Zimny D, Barg E. Down syndrome and hyperthyroidism-two case reports. Pediatr Endocrinol Diabetes Metab 2016 Feb 18;21(2):93-96.

11. Unachak K. Thyroid functions in children with Down's syndrome. J Med Assoc Thai 2008 Jan;91(1):56-61.

12. Rashid N, Mumtaz A, Mehmood S, Afzal N, Haq S, Ahmad T. Assessment of thyroid dysfunction in Down syndrome patients. Pak Paedr J 2009;33(4)211-217.

13. King K, O'Gorman C, Gallagher S. Thyroid dysfunction in children with Down syndrome: a literature review. Ir J Med Sci 2014 Mar;183(1):1-6.

14. Noble SE, Leyland K, Findlay CA, Clark CE, Redfern J. School based screening for hypothyroidism in Down's syndrome by dried blood spot TSH measurement. Arch Dis Child 2000 Jan;82(1):27-23. 